SLAC-PUB-10676

August 2004

\title{
The Definition and Classification of Universes*
}

\author{
James D. Bjorken \\ Stanford Linear Accelerator Center \\ Stanford University, Stanford, California 94309 \\ E-mail: bjorken@slac.stanford.edu
}

\begin{abstract}
We assume that the concept of a multiverse makes sense, and suggest a specific, standardized definition for member universes which are similar to our own. Central to this description is the definition of size, which is taken to be the asymptotic value, at large times, of the inverse Hubble constant. Thus the cosmological constant plays a central role in defining the properties of the subset of universes similar to our own. We then assume that vacuum parameters and coupling constants of the standard model are dependent upon the size of a universe, and propose a specific form for the dependence. Anthropic considerations then limit the size of habitable universes (as we understand that concept) to be within a factor two of our own. Implications of this picture for understanding the standard-model "hierarchy problem" are discussed, as are general issues of falsifiability and/or verifiability of these ideas.
\end{abstract}

Contribution to the proceedings of the symposium

"Universe or Multiverse?"

Sponsored by the John Templeton Foundation

Stanford University, Stanford, California

1, March 2003

*Work supported by Department of Energy contract DE-AC02-76SF00515. 


\section{Introduction}

When discussing the concept of multiple universes, it is a major challenge to keep the discourse within the bounds of science. There is an acute need to define what is being talked about. The issues include the following questions: How does one in general define a universe? Should one entertain different laws of physics in different universes? What are the most important parameters and/or features that characterize a universe? Once a parametrization has been attained, what is the differential probability of finding a universe with specified parameters? Is the integral of this distribution function finite or infinite?

A useful and familiar analogy is to consider Planet Earth as a universe. It is after all not so long ago that this was mankind's paradigm. Then one may take the ensemble of universes, or multiverse, to be the set of all compact massive objects within the solar system which orbit the sun-and/or each other. Alternatively one may take as a toy multiverse the set of all planets in the galaxy - or in the universe. In either case it is clear that the characterization of individual members of the ensemble is a very difficult task, and requires a sophisticated understanding of much of planetary science, especially the experimental side of the subject.

One simplification of the general problem of classification is to restrict the consideration to that subset of universes (or in the analogy, planets) which are nearly the same as our own. And this restriction can be naturally expressed in anthropic terms, by asking that the subset in question be that which admits in principle the existence of life as we know it. Even so, in the case of planets this restricted problem is still very difficult. It is not clear whether in our universe there is a large set of such planets, or whether the set only consists of our own planet. The problem is nicely described in the book "Rare Earth", by Ward and Brownlee [1].

If the corresponding problem for characterization of habitable universes within the multiverse is as complex as characterizing habitable planets within our universe, it is not at all clear that scientific methods will ever lead to noticeable progress in understanding. We can only hope that the case of universes is simpler than that of planets. In what follows we will assume that the characterization of universes can be made concise, and we will shape our further working assumptions with this in mind.

\section{What is a Universe?}

The characterization of universes naturally begins with the characterization of our own. In what follows, we shall adopt uncritically the standard model of contemporary cosmology. We assume that the spacetime geometry within the universe, as defined below in more detail, is spatially flat and can be described (in the large) by the Friedmann-Robertson-Walker (FRW) metric throughout its history. We assume that there is a nonvanishing cosmological constant, with dark energy comprising at present 70 percent of the energy of the universe, with most of the remainder contributed by 
dark matter. We also assume that prior to the ignition of the (radiation-dominated) Big Bang, there was an inflationary epoch characterized by a quasi-DeSitter spacetime, during which the FRW scale factor inflated by at least 30 powers of ten.

Strictly speaking, the size of such a universe is infinite, because the FRW spatial volume is infinite. But even if the FRW metric can be extrapolated to arbitrarily large distance scales, it still remains the case that almost all of this spacetime region is causally disconnected from us and will remain so to our descendants-provided the cosmological constant remains nonvanishing in the future (something which we will assume, for better or worse). So we place a box with comoving walls, centered on us, into the FRW spacetime. The dimension of the box at the present (FRW) time is, within a factor 10, taken to be of order ten times the nominal size of the universe, namely $30,000 \mathrm{Mpc}$, or $10^{29} \mathrm{~cm}$. For practical purposes we assume periodic boundary conditions at the surface of the box. Sensitivity of physical phenomena to this artifice can be tested by varying the (comoving) dimensions of the box. We do not expect significant sensitivity, because the box surface will be causally disconnected from us and our descendants.

Just as we bound by hand the spatial extent of our universe, we shall also bound the temporal extent. We choose as initial time a value for which the physical size of the comoving box is not too much larger than the Planck scale. Clearly, if the initial time were to be chosen much smaller, the size of the box would become small in comparison to the Planck scale, and the uncertainties in the underlying physics would grow considerably. And again there is good reason to believe that the phenomenology accessible to us is not strongly dependent upon details of the initial state which is assumed - although this question is still under vigorous debate.

It is also tempting to put a bound on the future as well as the past. Assuming the cosmological constant to be truly constant, there will be in the future a landmark time at about one trillion years, when the temperature of the primordial black-body radiation decreases to the Hawking temperature of our DeSitter spacetime, and almost all of the matter entropy has disappeared behind the DeSitter horizon. This occurs after the universe has inflated by a factor of about $10^{30}$ more than at present. Beyond this time the universe within the DeSitter horizon (the part causally connected to us and our descendants) is a truly quantum system, and again the uncertainties in the theoretical description are sure to be much higher. So we draw the line at this point.

In summary, we define our universe as the region of spacetime within the spacetime box defined above. Theoretical physics restricted to "inside the box" has a chance of being within the realm of physical science, i.e. verifiable or falsifiable by conceivable experiments. Outside the box it is much less likely that this is the case. I prefer to stay inside the box, and at most to compare the physics in our box with that in other hypothetical boxes, similarly constructed, located elsewhere in spacetime.

The most gross features of the universe "inside the box" can be best seen in the limit of ignoring small details such as the difference between the GUT and Planck scale, the difference between the initial Big Bang ("reheating") temperature and the 
Planck scale, and the very existence of ordinary and dark matter. Each of these simplifications involve replacing factors of $10^{5}$ or so by factors of order unity. While this is extreme, it is not a big deal in comparison with a remaining factor of $10^{30}$ in the description, which is ubiquitous. The history of this "universe in a box (for dummies)" divides itself into three epochs: quasi-DeSitter inflation, radiation-dominated Big Bang, and dark-energy-dominated DeSitter expansion. Each epoch is characterized by an increase in the FRW scale factor of about $10^{30}$. After the first epoch, the entropy of the radiation quanta accessible to our observation, and that of our progeny (of order $10^{90}$ ), is enclosed in a volume with dimensions of order millimeters, a scale of order $10^{30}$ larger than the Planck scale. And after the second epoch (nowadays, roughly speaking), the temperature of the primordial radiation is $10^{30}$ larger than the Hawking temperature of the future DeSitter spacetime, and is a factor $10^{30}$ smaller than the Planck temperature. All these factors of $10^{30}$ can be traced to the ratio of dark-energy scale to the Planck energy scale, i.e. to the value of the cosmological constant.

From this viewpoint, the cosmological constant is the most robust parameter characterizing the spacetime architecture of our universe and the subensemble of universes which have properties similar to ours. Indeed, we will define the size of a universe in these terms: it is the value of the inverse Hubble constant in the future, when dark energy overwhelms matter in the FRW evolution.

\section{Standard-Model and Cosmological Parameters in the Multiverse}

When considering the multiverse at large, it is possible that different universes have different gross histories. Do other universes go through inflation and radiationdominated Big Bangs? We shall assume that there is a subset which does so, in a manner similar to our own universe, and limit our attention to that subset. It is also possible that different universes have different laws of physics. For example, the pattern of symmetries in other universes may not be the same as what is expressed by the standard-model group; even the degrees of freedom might differ. Again, we do not here entertain such possibilities, but restrict our attention to those universes which have the same standard-model effective action as our own. However, we shall allow the parameters in that effective action to differ in different universes.

The candidate parameters which are necessary (but quite probably not sufficient) to specify a universe fall into different classes. One class of standard model parameters consists of those relevant to vacuum structure. This includes the scale of dark energy $(\mu=2 \mathrm{meV})$, of the QCD condensate $\left(\Lambda_{Q C D}=200 \mathrm{MeV}\right)$, of the electroweak vacuum condensate $(v=250 \mathrm{GeV})$, and perhaps of the grand unification (GUT) scales, ranging from $10^{13} \mathrm{GeV}$ (neutrino condensates?) to $10^{16} \mathrm{GeV}$ (coupling constant unification). Another parameter set consists of the magnitudes of the 
dimensionless coupling constants characterizing the strengths of the standard model forces. Another distinct set is the large number of parameters characterizing the masses and mixings of quarks and leptons (including neutrinos). Finally there is the set of parameters emergent from cosmological considerations, which may or may not be intimately associated with particle physics questions lying "beyond the standard model". These include the parameters defining the properties of dark matter, the baryon-to-photon entropy ratio, and the magnitude and spectrum of the temperature fluctuations of the primordial black-body photons.

If all of these parameters are independent, and determined at best by anthropic lines of argument, the description and characterization of universes will be very difficult. As promised in the preceding sections, we shall take a more optimistic point of view and assume that at least some of them are strongly correlated. Once one of the parameters in the correlated set is specified, the others are determined.

The material that follows is a brief summary of recent work based on this point of view $[2,3]$. Our starting point is the consideration of the vacuum parameters defined above. One of those parameters is the coefficient of the dark-energy term in the effective action. By definition, it is different in universes of different sizes, with size defined as in the previous section. As the size of a universe decreases, the dark-energy scale increases as the inverse square root of the size parameter. For Planck-sized universes, the dark energy reaches the Planck scale. For an idealized universe of infinite size, the dark energy would vanish.

We now make the basic assumption that the other vacuum parameters have a similar behavior. For example the QCD vacuum parameter $\Lambda_{Q C D}$ is assumed to vary as the inverse cube root of the size of the universe, and the electroweak condensate value $v$ as the inverse fourth root of the size. With this assumed behavior, all these vacuum scales converge, or flow, toward a fixed point which is of order Planck/GUT scale in energy, when the size of the universe becomes of order the Planck/GUT size.

(A caveat: the assumed dependence of standard model parameters with size is with respect to the time-independent cosmological constant, not the FRW scale factor. We do not entertain here time-dependence of standard-model parameters within our own universe, but only compare the parameters in different universes. However, a possible exception is mentioned in the last paragraph of this note.)

Given this assumption, more can be deduced. The dimensionless strong coupling constant $\alpha_{s}$ of QCD is determined by $\Lambda_{Q C D}$. It is a "running coupling", dependent upon the momentum scale probed. Since the size dependence of this running coupling constant is determined, this is also true when the coupling constant is evaluated at the GUT scale, where it is presumably "unified" with the electroweak couplings, which also "run". Therefore the size dependence of electroweak and electromagnetic coupling constants at low energy scales is also determined. The result is that the inverse "fine structure constants", including the famous 137 of quantum electrodynamics, depend linearly on the logarithm of the size of the universe, and vanish for small Planck/GUT-scale universes. This means that the standard model forces are 
very strong and nonperturbative for such small universes, and vanish in the limit of an infinite universe. What is strongly suggested is that an infinite universe, as we have defined it, is nearly trivial, containing no standard-model interactions whatsoever other than gravity.

It is also reasonable, albeit more uncertain, to expect that the mass parameters for the quarks and leptons follow a similar pattern, i.e. they flow to values of order the Planck/GUT scale for small universes and tend to zero for very large universes. But the details become increasingly fuzzy as the masses become small. In particular the largest uncertainties occur for the anthropically significant masses, such as the electron mass and the up- and down-quark masses, important for the details of nuclear physics that condition our existence. It is the lack of theoretical understanding of the basic origin of these small masses which is the roadblock.

\section{Anthropic Considerations}

For better or worse, the scaling rules enunciated above allow detailed study of the physical properties of universes with sizes different from our own. This is the main content of the aforementioned reference [2]. What is found is that the existence of chemistry is robust; the size of the universe can be varied by 30 powers of ten without major effects. In broad terms the same is true for nuclear physics. However, as is wellknown to the anthropic community, there are details, essential for the existence of life as we know it, which are not robust. Examination of the anthropic constraints shows that, in the context of our assumed scaling rules, the strongest limitation on the size of universes which can support life as we know it comes from the famous triple- $\alpha$ process responsible for the synthesis of carbon in stars. The overall strength of the nuclear force cannot vary by more than a fraction of a percent without causing trouble. In the case of interest this variation is effected only via chiral symmetry breaking, i.e. by the nonvanishing masses of the up, down, and perhaps strange quarks. Those mass

parameters have a different dependence on the size of the universe than does $\Lambda_{Q C D}$, and it is this disparity which destroys the delicate balance of parameters which allows the triple-alpha reaction to proceed.

When the dust settles, the bottom line is that the size-variation allowed by the existence of life as we know it is of order of a factor two. In a universe twice as large or twice as small as ours, the standard-model parameters would arguably be different enough to block the production of carbon in stars, and hence block the evolution of life as we know it. The above estimate is quite uncertain-perhaps off by a factor three or even ten. But it is accurate enough to draw a variety of tentative inferences. The most important inference has to do with the "hierarchy problem", and it provides an a posteriori reason to take the scaling assumption seriously.

What is generally denoted by the hierarchy problem is the large disparity between the electroweak scale, characterized by the vacuum parameter $v$, and the Planck/GUT scale. However it also includes the notorious "cosmological constant problem": why 
the cosmological constant scale is 30 powers of ten smaller than the Planck/GUT scale. In addition there is the "problem of mass", which includes the issue of why the electron mass, say, is so much smaller than the top-quark mass. If the scaling behavior is assumed, all of these questions are rendered moot. For small universes, there is no hierarchy problem; all these parameters arguably take values of order the Planck/GUT scale. And it may well be the case that the typical universe is in fact small. It is only because we live in such a large universe that we see these huge hierarchies of scale. And the above anthropic considerations require us to live in a large universe; the conditions of life as we know it only exist for large universes.

While this argument falls short of a full resolution of the hierarchy problem, it does provide a different way of viewing it. The problem of divergences and renormalization, which is part of the usual statement of the problem, is now expressed as the question of why the renormalized vacuum parameters should be dependent upon the size of the universe, and in the specified way. In other words, one must understand the scaling exponents such as $1 / 3$ and $1 / 4$ for strong and electroweak sectors, respectively. This author has some ideas about the $1 / 3$. Others, in particular Tom Banks, have already speculated about the $1 / 4[4]$.

\section{The Distribution Function for Universes}

The above considerations embolden us, perhaps foolishly, to speculate on the question of the size distribution of universes in the ensemble, or subensemble, we have been looking at. It seems most reasonable to assume that the total number in the ensemble is finite. It also seems reasonable to assume, given the hierarchy arguments of the previous section, that the distribution peaks at small sizes, of order Planck/GUT size. Given all that, the remaining question is the asymptotic behavior at large sizes. Two natural classifications are power law and exponential. Anthropic considerations argue that the integral of the distribution over the habitable interval should give a number large compared to unity, in order to make the universes which are in principle habitable (as we understand the term) non-unique. For any reasonable falloff with size, such an estimate gives essentially the same result as integrating over all sizes as large or larger than our own. And it is also clear that if the falloff is exponential or faster, the total number of the universes in the sample must be gigantic, e.g. of order $10^{10^{60}}$. Modesty therefore suggests the alternative choice of a power-law tail. If the distribution function $R d N / d R$ falls off at large sizes $R$ as $R^{-n}$, the total number of universes in the ensemble will be bounded below by a number of order $10^{60 n}$. This is a big number to be sure, but not very far beyond other big numbers encountered in the study of our own universe. In addition, power-law behavior is often associated with the notion of criticality, and/or scale-invariant behavior. The feature of spatial flatness of our universe may suggest criticality as an underlying feature of the future, better theory, and/or of the subset of universes with features similar to our own.

It is unrealistic to expect the ensemble of universes to be characterized by only 
one parameter, the size. It is therefore of interest to look at the remaining candidate parameters and search for those most likely to be "independently anthropic". Amongst the standard model candidates, the light-quark (up and down) masses are strong ones, as forcefully advocated recently by Craig Hogan [5]. And at least some subset of the three cosmological parameters mentioned in Section 3 seem to be strong candidates as well, in order that there occur the right amount of large-scale structure in our universe. However, we do not have anything very new to add to this problem. Better understanding of the nature of dark matter would be of obvious help.

\section{Concluding Comments}

It should be abundantly clear that the above discussion skirts dangerously close to the edge of legitimate science. Are any of these speculations falsifiable or, even better, verifiable? With regard to falsifiability, there is an answer: if the cosmological constant is eventually found to be zero, the scaling ideas die an unambiguous death. Likewise if the cosmological constant is not constant, and exhibits a lot of quintessence, it could well be that the implied time variation in other standard model parameters would exceed experimental limits. More interesting is the question of verifiability. Probably the best chance lies in finding a microscopic theory consistent with the scaling rules which has predictive power above and beyond what we now have. As mentioned in Section 4, there are some reasons for optimism in this regard.

It would also be advantageous if individual universes were in causal contact, which might admit experimental investigation, at least in principle. This is unlikely, and goes against the grain of almost all contemporary thinking. But it is perhaps not completely out of the realm of possibility. A few individuals, including this author, now and then entertain the notion of black hole interiors being non-singular static DeSitter space, as is the case to good approximation for our own universe. This invites a model of the multiverse as nested black holes, with the remote possibility of two-way communication through the horizons.

Another possibility is that the universe-in-a-box defined in Section 2 really consists of two universes. The first one is the inflationary universe present before ignition of the Big Bang. It is characterized by a huge cosmological constant (in the approximation of "no-roll" instead of slow-roll). Perhaps the standard model parameters should take the values appropriate to the interpretation of that piece of spacetime as a "small universe", with size parameter (Hubble scale) of order $10^{13} \mathrm{GeV}$ (or $10^{-27} \mathrm{~cm}$ ). It is interesting that, were this to be done, the dark-energy scale is of order the GUT scale, with the QCD and electroweak vacuum energy scales, naively estimated from the power-law rules, somewhat higher. It is easy to imagine that in fact these three scales become synthesized, and that the interpretation of the inflaton field could be in terms of QCD and/or electroweak condensates, which in turn might be more appropriately re-interpreted as a GUT condensate. The Big Bang would then be ignited by the "decay" of the "small" universe into our "big" universe, accompanied somehow by a 
large amount of entropy production. This idea has not yet been pursued in detail. But the risk is not that this line of thinking has no phenomenological consequences, but rather that it has too many.

\section{References}

[1] D. Brownlee and P. Ward, "Rare Earth" (Copernicus, Springer-Verlag, New York, NY, 1999).

[2] J. Bjorken, hep-th/0210202.

[3] For elaboration of the material presented here, see J. Bjorken, astro-ph/0404233.

[4] T. Banks, hep-th/0206117.

[5] C. Hogan, astro-ph/9909295. 